\title{
Les stratégies d’ingénierie du tissu osseux : le présent et le futur...
}

\author{
Amédée J \\ Inserm U577, Université Victor Ségalen, Bordeaux, France \\ joelle.amedee@inserm.fr
}

L'extraordinaire augmentation de la longévité humaine justifie des besoins croissants en organes de remplacement. Les succès remarquables obtenus avec les transplantations classiques comportent trois inconvénients principaux : pénurie du nombre de greffes disponibles, risque significatif de rejet et transmission d'agents pathogènes.

Pour pallier les limites de ces stratégies de réparation, de nombreux travaux sont consacrés, dans la littérature, au développement de nouveaux biomatériaux innovants et de nouveaux concepts de réparation pour la médecine régénératrice appliquée au tissu osseux. De façon plus précise, pour permettre aux matériaux de mieux s'intégrer au sein des tissus receveurs, le concept d'ingénierie tissulaire prévoit d'associer à la composante artificielle d'origine synthétique ou naturelle une composante cellulaire ou tissulaire d'origine autologue. Ces matériaux "hybrides" seront ainsi aptes à participer à la néoformation des tissus à réparer et/ou à assurer la fonction de l'organe à substituer. Il s'agit là d'un champ de recherche et de biotechnologie qui combine divers aspects de la biologie cellulaire et moléculaire, et de la science des matériaux. Ainsi, l'ingénierie tissulaire tire partie entre autres des avancées réalisées par les spécialistes de la biologie du développement et des cellules souches (embryonnaires, adultes), et les spécialistes des biomatériaux, principalement orientés vers le développement de nouvelles matrices tridimensionnelles innovantes.

Une autre évolution dans le domaine de l'ingénierie tissulaire relève du progrès des connaissances fondamentales sur l'importance des mécanismes d'interactions et de communications cellulaires que peuvent établir les cellules sollicitées dans un processus de réparation de tissu. De façon plus précise, le rôle clairement identifié de la vascularisation dans la néoformation osseuse a permis d'identifier de nouvelles méthodes de reconstruction du tissu osseux en faisant appel à des techniques de pré-vascularisation in vitro ou in vivo des biomatériaux de substitution osseuse Le développement de ces stratégies, une fois maîtrisées, le choix du support 3D, le choix et l'exploitation du matériel cellulaire, devraient déboucher sur de nouvelles familles de produits, aux côtés des médicaments, des dispositifs médicaux, et des transplantations de tissus ou d'organes (avec leurs limites et leurs risques) dont médecins et chirurgiens pourront faire bénéficier les patients pour l'amélioration de leur qualité de vie. 